\title{
A Novel Honeycomb Routing Approach for Bandwidth Utilization in MANET
}

\author{
O. Cyril Mathew*, A. M. J. Md. Zubair Rahman \\ ${ }^{1}$ Anna University, Chennai, India. \\ 2 Al-Ameen Engineering College, Erode, India.
}

Corresponding author. Email: mathewphd@gmail.com

Manuscript submitted January 6, 2015; accepted April 8, 2015.

doi: 10.17706/jsw.10.6.659-669

\begin{abstract}
Manet is an independent and distributed system of mobile nodes that are connected by means of the wireless link. Exploiting resources in a systematic manner without wastage would increase the throughput of the network. Bandwidth is one of the vital resources in the network traffic. Bandwidth generally refers to the data rate at which a network path or link transfers a packet. It is one of the guarantee features for measuring the network performance. Moreover, the bandwidth of the MANET is insufficient than that of the wired network. In the meantime, bandwidth exhaustive wireless applications are emerging that influences the bandwidth demand on the wireless network. Even if advancements have been made in the high speed wireless channel, bandwidth is yet a challenging concern because of the limitations of the wireless channel. Hence, an effective utilization of bandwidth is necessary for improving the network performance. Thus, a novel Honeycomb Routing (HCR) algorithm from the two-dimensional Honeycomb structure, has been proposed in this paper in order to effectively utilize the bandwidth of the network through the Adhoc on-demand distance vector (AODV) routing protocol with the admission control algorithm in MANET. This approach provides a high bandwidth which is obtained by sharing the links and the successful flow of data packets by using the wait and bound technique in the admission control algorithm. Simulations are carried out to analyze the performance of the proposed approach.
\end{abstract}

Key words: Mobile adhoc network, adhoc on-demand distance vector, honeycomb routing, quality of service, wait and Bound.

\section{Introduction}

The mobile adhoc network is a group of wireless mobile nodes [1], [2] in which node cooperate with each other by transmitting packets to enable communication with a node. The data packets pass through more than one intermediate node that acts as a router as well [3], [4]. Because of their low signal power, restricted bandwidth and high mobility of nodes, frequent link breakage occurs, thereby resulting in the establishment of fresh links. Because of this dynamic nature, the routing protocols should be designed in a manner that is adaptable and maintain routes within the network. However, it introduces the considerable issue in the routing algorithms.With routing the information about the nodes aids in identifying the link between the sender and the receiver, minimizes the delay in transmitting the data. 
The effective interconnection networks have significant impact on the communication performance during the exchange of messages. Especially in packet networks, the bandwidth refers to the volume of data transmitted over a link [5]. In considering the multimedia applications such as live streaming of audio or video data file, the available bandwidth impacts the performance of the application directly. A high bandwidth link ensures high throughput and lower end-to-end delay. Hence, Bandwidth plays a very important role in various network technologies and its estimation is vital to ensure QoS in wireless networks.

In this paper, honeycomb routing (HCR) algorithm based two-dimensional Honeycomb structure has been proposed which provides the broadcasting information of the data packets between the nodes in the interconnection networks. The AODV routing mechanism in HCRalgorithmaids in route establishment using RREQ (route request) and RREP (route reply) control messages. Each node checks its routing table to ensure whether it contains a route to the destination. If the route does not exist, the RREQ message is then broadcasted to its neighbors. In this routing protocol, each node of the network maintains routing tables that are periodically updated by sending a broadcast message to the entire network by using HCR algorithm. During broadcasting the data packets may result in dropping, to overcome this dropping an Admission control algorithm is used [6].The wait and bound technique in the Admission control algorithm provides a proper flow of data packets without loss of data, thereby results in increasing the effectiveness of Bandwidth in MANET.

The remainder of the paper is as follows: in Section 2 previous research work carried out in bandwidth utilization has been presented. Section 3 presents the network model of the proposed approach for effectively utilizing the bandwidth. In Section 4 simulation results are presented and finally Section 5 concludes the paper.

\section{Related Work}

The author [5] proposes a strategy for effectively utilizing the bandwidth. This strategy uses the notion of virtual circuit (VC) approach in wireless network which has been dealt with several recent research works. In this strategy the bandwidth utilized by the header is significantly reduced and that bandwidth is utilized by the data/payload. But, a drawback of this approach is that the virtual circuit has to be reestablished if a mobile node moves away.

In the paper [7] utilized the concept of a dynamic stipulating architecture for firmly distributing bandwidth depending on it effectiveness. The essential bandwidth is initially allocated based on the requirement of the user and the broadcast is monitored in order to change the allocation of bandwidth if some bottleneck occurs. Moreover, the network intermittently identifies the unexploited bandwidth and allows user who require extra bandwidth to utilize that bandwidth to transfer packets at a higher rate.

In [8] priority scheduling has been carried out in wireless ad hoc networks have been performed in order to fully utilize the available bandwidth, reducing the packet loss incurred due to nodes mobility. In this approach two exclusive busy tone packets that specify the packet transmission by a high priority nodes have been utilized thereby determining the channel contention. The piggy banking approach employed here uses the channel effectives that lets the high priority data to assist the low priority data by distributing the unexploited bandwidth.

Ref. [9] dissected the work of the utilization of bandwidth has been increased based on the priority with the zones in the wireless environment. The zones are set based on the range and demands of the user. The desired needs of the user within the zone bandwidth range are allocated to the user. The resources are reallocated when desired by the other users. Based on this zone based protocol the requirements of the user are satisfied. 
Ref. [10] Presents an optimal bandwidth allocation approach for IEEE 802.11e MANET. In this approach, the residual bandwidth is computed by utilizing the bandwidth probing approach and the measured bandwidth is shared between reserved and shared regions utilizing bandwidth sharing techniques for both real-time and non-real time traffic flows. The bandwidth allocation policy, then allocates the extra bandwidth in the reserved region of the host that requires additional bandwidth.

In [6] integrated the QoS (Quality of Service) with routing processes and bandwidth estimation have been done by distributing the bandwidth information using hello message.

Here, an admission control and feedback scheme has been implemented by employing hello bandwidth estimation and listen bandwidth estimation techniques. In this; the first neighbor's relay is utilized to obtain the information about the second neighbor. Once, the node receives the update, they are disseminated to the next hop neighbor. Based on this information packets are forwarded to the neighbors.

In [11] a hypothetical model for computing the path's residual bandwidth has been developed that considers both background traffic and traffic along the path. In [12] presented a system model for QoS oriented bandwidth calculation, where the scheduling at the MAC layer has been changed and the slots have been set to transfer data, thereby increasing data rate and increasing the utilization of bandwidth.

\section{Proposed Methodology}

\subsection{Network Model}

In this section, the effective bandwidth utilization in MANET is described by considering a honeycomb network. In the honeycomb tessellation, every cell contains six neighbors covering the environments from all the directions. For any two adjacent cells, each node in one cell communicates with all the nodes contained in another cell. Here, a two dimensional honeycomb structure is utilized as shown in Fig. 1.

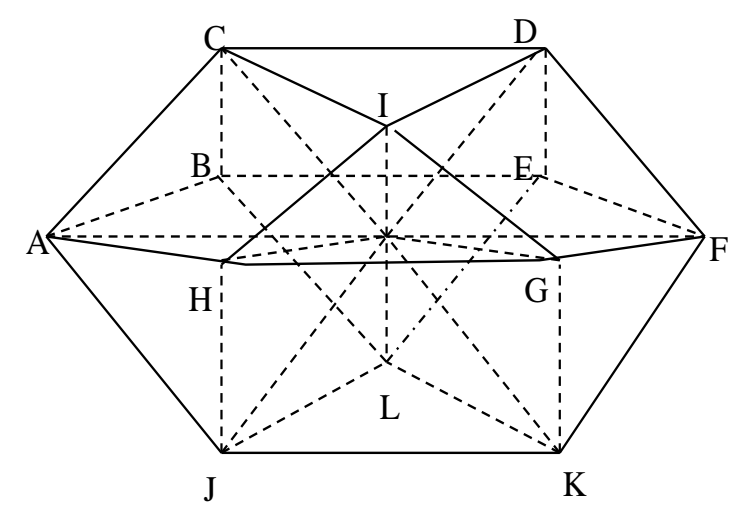

Fig. 1. A typical two-dimensional honeycomb structure of size 3.

The two dimensional honeycomb structure is built utilizing tetrahedron as follows:

A tetrahedron center is joined with the four neighboring tetrahedrons center, where each tetrahedron distributes one of its surfaces with the first tetrahedron. The centers are considered as the processors of the 2D honeycomb, with edges connecting centers of the two neighbors. This procedure is iterated for every newly inserted tetrahedron. As in 2D structure, links could be viewed as vectors, and to describe a 3D honeycomb, we require four of them. Every node of the 2D honeycomb can be defined utilizing four unit vectors as the coordinate axis. The Bandwidth of the link is the total amount of information that can be transferred in a certain period of time at a particular rate. Let the bit rate of the packet be $\tau$ kilobits per second and bandwidth of the link be $\beta$ kilobits per second. Generally $\tau \leq \beta$, since packets cannot be placed on the link at a rate larger than that of the bandwidth of the link. If a packet with a bit rate greater than that 
of the bandwidth of the link ( $\tau>\beta$ ) is placed on the link, only $\beta$ bits of the packets would be transmitted and remaining $(\tau-\beta)$ bits will be discarded.

\subsection{Honeycomb Routing Algorithm in AODV Protocol}

In order to overcome the above issue the Honeycomb Routing (HCR) proposed in this paper effectively utilizes the link bandwidth capacity. In this framework, the link along the path between the source node and the target node is assumed to have a bandwidth of $\beta$ (kbps).The packets arriving for transmission on the link are carried through the Adhoc on-demand distance vector (AODV) protocol. When a node needs to transfer packets to other node, a Route Request (RREQ)Packet is broadcasted as shown in Fig. 2. The RREQ packet contains the source and the destination address, destination sequence number and desired bandwidth. Upon receiving the RREQ packets; the node compares the desired bandwidth $\left(\beta_{\text {des }}\right)$ with the residual bandwidth of the link $\left(\beta_{\text {res }}\right)$. With a generic link i and bandwidth capacity $\beta_{i}$, the residual bandwidth $\beta_{\text {res }}$ is given by

$$
\beta_{\text {res }}=\beta_{i} \times\left(1-c_{i}\right)
$$

Here, $c_{i}$ utilizes the bandwidth of the link. The source transmits the data to the destination if the desired bandwidth is less than the residual bandwidth of the link $\left(\beta_{\text {des }}<\beta_{\text {res }}\right)$.

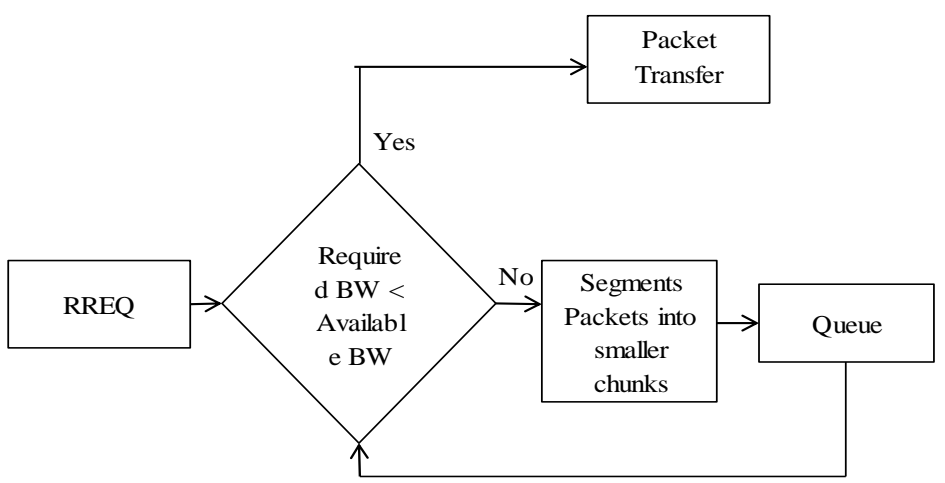

Fig. 2. Packet transfer in honeycomb routing.

The network consisting of a set of nodes ( $n i, n j$ ), where $f$ denotes the frequency of node $i$ and $j$. The broadcasting transmission ( $t x i$ ) of data packets in AODV occurs between the nodes $i$ and $j$. consider a network topology ' $T$ ' which is of mxn dimension.

\section{Pseudo code for HCR algorithm for packet transfer in AODV}

The nodes get initialized and they are randomly arranged in a network and undergo broadcasting condition.

BCST condition:

For all $n(i)$ that belongs to $T$,

L1:

Check

If $n i[x, y] \sim n j[x, y]<$ bcst range then

initialize bcst from $n(i), n(j)$

$\operatorname{read} f(i), f(j)$

if $f(i)=f(j)$ then

increase mobility of $n(j), n j[x, y]=$ new $n j[x i, y i]\{x !=x i$ and $y !=y i\}$

check 


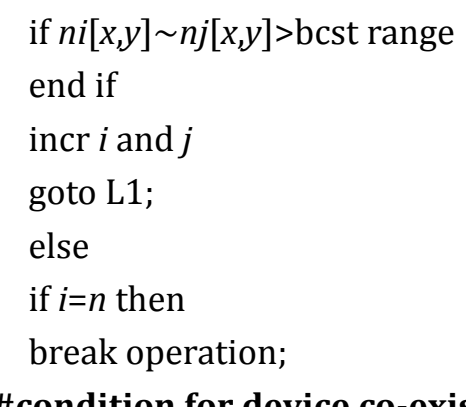

\section{\#condition for device co-existence}

Read $t x(i)$ and $t x(j)$

If $t x(i)<\operatorname{sum}$ of all $\{t x$ from $j$ to $n\}$ then

Next node $(i)=n(j)$

Else

increase mobility of $n(j), n j[x, y]=$ new $n j[x i, y i]\{x !=x i$ and $y !=y i\}$

check

if $n i[x, y] \sim n j[x, y]>$ bcst range

end if

repeat through $L 1$ for all $i$ to $n$

end if.

$n(i), n(j) \rightarrow$ node ' $i$ ' and ' $j$ '

ni $[x, y] \rightarrow$ coordinates of node $i$

$n j[x, y] \rightarrow$ coordinates of node $j$

$n i[x i, y i] \rightarrow$ new $x, y$ coordinate position of ' $i$ ' node (after movement)

$n j[x i, y i] \rightarrow$ new $x, y$ coordinate position of ' $j$ ' node (after movement)

$f(i), f(j) \rightarrow$ frequency of ' $i$ ' and ' $j$ '

$t x(i) \rightarrow$ transmission of ' $i$ ' in the given $f(i)$

\section{\#\#Routing Process}

For all 1 to $n$, construct Routing Tree

RT_UPDATE $=$ Nil

if $w t(i)<w t(j)$ then

neighbor $=n(i)$

if(seq_num ${ }_{i}<$ seq_num $\left._{j}\right) \|\left(\right.$ seq_num $_{i}=$ seq_num $\left._{j}\right) \& \&\left(\right.$ hop_count $_{i}<$ hop_count $\left._{j}\right)$

then

(seq_num ${ }_{i}=$ seq_num $_{j}$ );

hop_count $t_{i}=j+1$;

end if

call HC1 \#\#above pseudo code

else neighbor $=n(\mathrm{j})$

increment $I, j$ to $n$.

end if

where $w t(i) \rightarrow$ weight of ' $i$ ' node, $w t(j) \rightarrow$ weight of ' $j$ ' node, seq_num ${ }_{i}$,Seq_num ${ }_{j} \rightarrow$ sequence number of nodes $i, j \ldots . . . .$.

AODV route update in honeycomb, yields loops free routes. If a loop is formed along the route to destination $\mathrm{d}$ whose size is $n\left(l_{1}, l_{2}, \ldots . ., l_{m}, l_{1}\right)$, which can be expressed as

$$
\operatorname{seqnum}_{i}^{d} \leq \operatorname{seqnum}_{j}^{d}
$$


Then, the following conditions should be true between the nodes of the loop, which implies

$$
\text { seqnum }_{i 1}^{d} \leq \text { seqnum }_{i 2}^{d} \leq \text { seqnum }_{i n}^{d} \leq \text { seqnum }_{i 1}^{d}
$$

This implies,

$$
\text { seqnum }_{i 1}^{d}=\text { Seqnum }_{i 2}^{d}=\text { Seqnum }_{i n}^{d}=\text { Seqnum }_{i 1}^{d}
$$

Route maintenance is performed by using route error $\left(R_{E R R}\right)$ packets. When an intermediate node identifies a link failure, a RERR error message is generated and forwards it to the source if the residual bandwidth is less than the desired one $\left(\beta_{\text {des }}>\beta_{\text {res }}\right)$. The source node upon receiving the error message divides the data packets into small chunks in order to avoid over utilization of the bandwidth and these packet chunks are transmitted in succession. The RERR message proliferates towards the source node through the failed link and removes all the damaged routes. Except from route maintenance AODV employs a timer based approach to eradicate the sale routes. The admission control algorithm is used to achieve this.

\subsection{Admission Control Algorithm}

For any node, estimating the residual bandwidth is appropriate to take a control decision. For calculating the available bandwidth, each source adapts its admitted traffic effectively by limiting the amount of data traffic. So throughput is consistent, delay and packet loss is minimized for all acknowledged flows. Let $\alpha$ represent the data generation rate of a node and $\alpha_{i}$ represents $\alpha$ at the $i^{\text {th }}$ index of the averaging window. We further assume that $\rho$ represents the channel rate, $\omega$ represents the average available bandwidth in kbps and $\mu$ denotes the link bandwidth. Therefore, at each bandwidth measurement instance, the available bandwidth is calculated by using the average data generation rate on nodes within the interference of the node using Equation (5).

$$
\omega=\rho-\left(\sum_{\mu=i}^{0}\left(\left(\alpha_{\mu}+\beta_{r e s}\right)+R_{E R R}\right)\right) k b p s
$$

When the next data packet request for service, then the following checks are performed:

1) Determining whether the overall bandwidth utilization is within its capacity by employing the eq (6) and check that the restrained bandwidth for the assured service flows does not exceed the capacity of the link.

$$
\mu=r^{a}+R_{G}
$$

Here $r^{a}$ represents next sequence of data packets for transmission and $R_{G}$ is the reserved rate of guaranteed flow of data packets.

2) Determine that if all guaranteed flow of data packets use their desired bandwidth, the network will still able to meet its guaranteed service delay bound remains constant, which is given by

$$
\text { Delay }=\frac{r^{a}+R_{G}}{\beta_{d e s}-\mu}
$$

The wait and bound Technique are used to send the data packet particular with the time period to overcome the delay problem.

\subsection{Wait and Bound Approach}


A packet arriving at the link has to wait until the previous packet has been successfully transmitted. The packets that are waiting for the link are lost after a particular time period ti secs. Hence, to avoid the packet loss, these packets are stored in the queue maintained in every node as illustrated in Fig 3. The queue is FIFO with a simple priority. Once, the packets are divided into smaller chunks, the source broadcasts the RREQ packets for every packet chunk placed in the queue. The packets in the queue are served using a technique [2]. Fig. 3 illustrates the packet transmission process by effectively utilizing the bandwidth. In wait and bound technique each packet in the queue is assigned a particular time quantum. If the queue is not able to transmit the packet at the particular time quantum, those packets are placed at the tail of the queue and transmitted during next time quantum. Fig. 4 shows the process of packet transmission by using the wait and bound.

Queues in the nodes to avoid packet loss
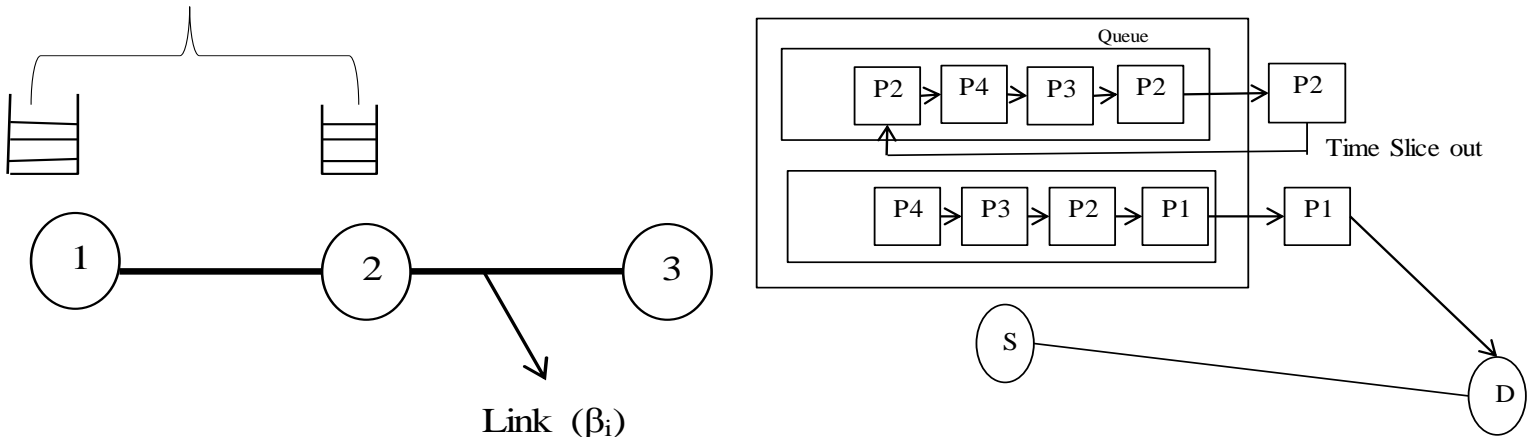

Fig. 3. Nodes with queue to avoid packet loss. Fig. 4. Illustrations of wait and bound approach.

Upon receiving the RREQ the destination node performs the comparison check and allows for packet transmission. The process is repeated until the entire packet has been successfully transmitted and it results in effective bandwidth in the network.

\section{Simulation Results}

\subsection{Simulation Setup}

Simulations are carried out to analyze the performance of the HCR approach. Here, Network Simulator 2(NS2) is used as simulation platform. The network comprises of 50 mobile nodes that are dispersed randomly over an area of $1500 \times 1000 \mathrm{~m}$ area. The simulations are carried out employing Ad hoc on demand distance vector routing protocol .Here, the bandwidth capacity of the link is considered as $10 \mathrm{Mbps}$ and the basic data rate is taken as $1 \mathrm{Mbps}$. The parameters that are considered for performance evaluation is shown in Table 1 respectively.

\subsection{Performance Metrics}

Delivery ratio: The total number of packets arrived at the intended destination node to the total number of packets that has been sent by source.

$$
\mathrm{DR}=\frac{\text { Number of packets succesfully received }}{\text { Total number of packet sent }}
$$

Delay: The delay is time taken by the packet to reach the destination and is given by

$$
\text { Delay }=\frac{\sum_{i=1}^{N}\left(p_{r}^{i}-p_{s}^{i}\right)}{\text { No of packets received }}
$$


Table 1. Simulation Parameters

\begin{tabular}{|l|l|}
\hline \multicolumn{2}{|l|}{ Simulation and Network Parameters } \\
\hline Network Area & $1500 \times 1000$ \\
\hline Routing Protocol & AODV \\
\hline No. of Mobile Nodes & 50 \\
\hline Network Topology & Flat Grid \\
\hline IEEE Standard & 802.11 \\
\hline Broadcasting Range & $550 \mathrm{mts}$ \\
\hline Application Type & Cbr \\
\hline Application rate & $1.0 \mathrm{mb}$ \\
\hline No. of Packets & 1500 \\
\hline Simulation Time & $50 \mathrm{~s}$ \\
\hline Data Transfer Protocol & TCP/ UDP \\
\hline Connection Type & One-to-one \\
\hline Bandwidth & $10 \mathrm{Mbps}$ \\
\hline Basic Data Rate & $1 \mathrm{Mbs}$ \\
\hline
\end{tabular}

Here, $p_{r}^{i}$ is the packets received at the time $\mathrm{t}, p_{s}^{i}$ is the packet sent at the time $\mathrm{t}$ and $\mathrm{N}$ is number of packets received.

Throughput: It is defined as the total number of bits delivered by total duration of simulation time. It is a measure of packets that are successfully delivered to a final destination sent from the sender. It shows how effectively the requests are handled.

$$
\text { Throughput }=\frac{\text { Total number of packets sent }}{\text { simulation time }}
$$

Bandwidth: It is defined as the average rate of successful data transfer through a communication path and it is given by,

$$
b_{w}=\sum_{j \in N(i)} b_{j}
$$

where $N(i)$ is the number of nodes, $b_{j}$ is the bandwidth consumed by all the connections of node $j$. If the total bandwidth is $b_{\max }$, then the available bandwidth is computed as,

$$
B=b_{\max }-b_{w}
$$

Table 2. Bandwidth Utilized by TSP and HCR

\begin{tabular}{|l|l|l|}
\hline No. of Packets & $\begin{array}{l}\text { Bandwidth Utilized } \\
\text { (Kbps) in case of } \\
\text { TSP }\end{array}$ & $\begin{array}{l}\text { Bandwidth Utilized } \\
\text { (Kbps) in case of } \\
\text { Proposed approach } \\
\text { (HCR) }\end{array}$ \\
\hline 1000 & 5.1147 & $\mathbf{5 . 4 3 2 8}$ \\
\hline 1500 & 5.8472 & $\mathbf{7 . 9 2 5 6}$ \\
\hline 2000 & 7.1204 & $\mathbf{8 . 0 0 3 7}$ \\
\hline 2500 & 7.5344 & $\mathbf{9 . 1 2 5 4}$ \\
\hline
\end{tabular}

\subsection{Results and Discussions}


The Fig. 5 illustrates the comparison of honeycomb routing approaches with the TSP(Travelling Salesman Problem) and Table 2 shows the amount of bandwidth utilized by the TSP and HCR approach. The number of packets, delivered is high on HCR approach with the corresponding effective bandwidth efficiency (91\%) whereas in TSP results in lesser transfer of data packet yielding a lower bandwidth of (75\%).

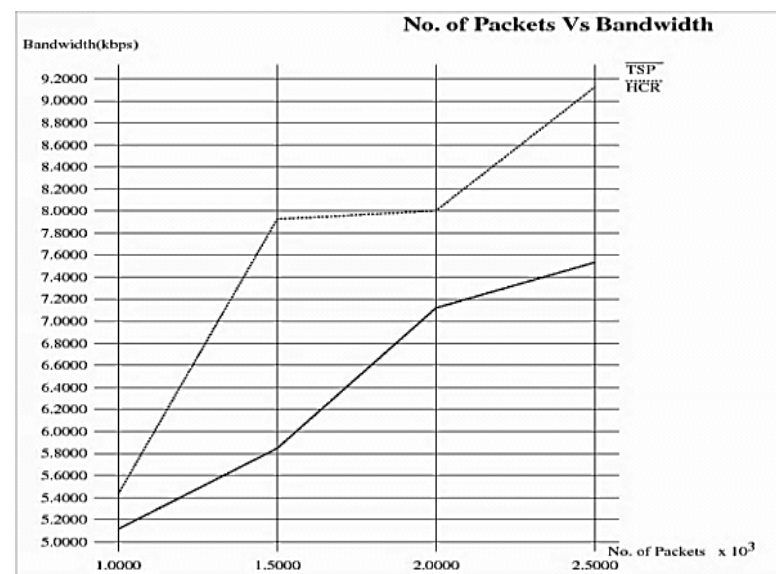

Fig. 5. No of packets VS Bandwidth.

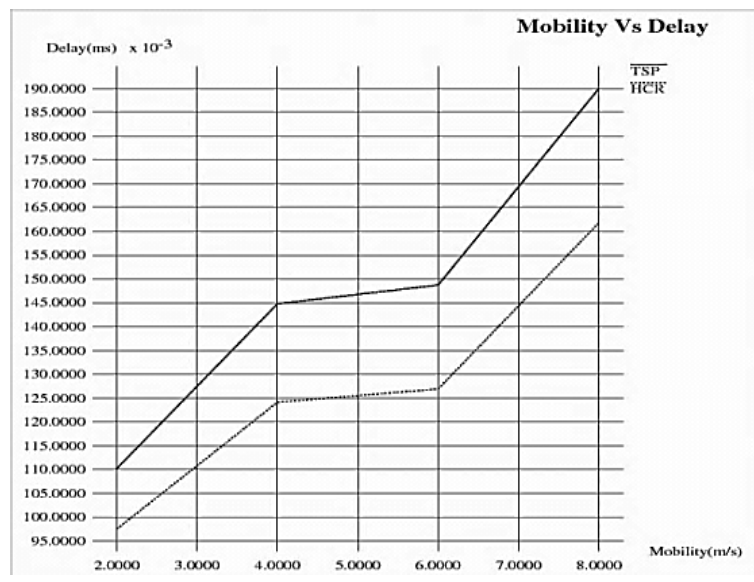

Fig. 6. Mobility VS delay.

Table 3. Delay in TSP and HCR

\begin{tabular}{|l|l|l|}
\hline Mobility & Delay (ms) incurred by TSP & $\begin{array}{l}\text { Delay (ms) incurred by Proposed } \\
\text { approach (HCR) }\end{array}$ \\
\hline 2 & 0.110 & \\
\hline 4 & 0.144 & $\mathbf{0 . 0 9 7}$ \\
\hline 6 & 0.148 & $\mathbf{0 . 1 2 4}$ \\
\hline 8 & 0.192 & $\mathbf{0 . 1 2 6}$ \\
\hline
\end{tabular}

Table 4 Delivery Ratio of TSP and HCR

\begin{tabular}{|l|l|l|}
\hline Mobility & Delivery Ratio (\%) of TSP & $\begin{array}{l}\text { Delivery Ratio (\%) of the Proposed } \\
\text { approach (HCR) }\end{array}$ \\
\hline 2 & 66.24 & $\mathbf{7 2 . 4 5}$ \\
\hline 4 & 61.11 & $\mathbf{6 8 . 2 9}$ \\
\hline 6 & 57.84 & $\mathbf{6 1 . 5 7}$ \\
\hline 8 & 53.06 & $\mathbf{6 1 . 0 7}$ \\
\hline
\end{tabular}

The Fig. 6 illustrates the minimum delay time vs mobility in Honeycomb routing by comparing with the TSP and Table 3 shows the delay occurred in both TSP and HCR approach. The mobility signifies the node's movement in a network, which depicts the delay time. Thus the delay time in HCR is reduced $(157 \times 10-3 \mathrm{~ms})$ comparing to the TSP, and the bandwidth efficiency has increased to a maximum extent due to the minimized delay time.

The Fig. 7 illustrates the delivery ratio in Honeycomb routing by comparing with the TSP and Table 4 shows the delivery ratio obtained in case of TSP and HCR approach. The delivery ratio in broadcasting the number of packets is high (72.5\%) whereas in TSP results in lesser transfer of data packets yielding a lower delivery ratio $(66.5 \%)$. 


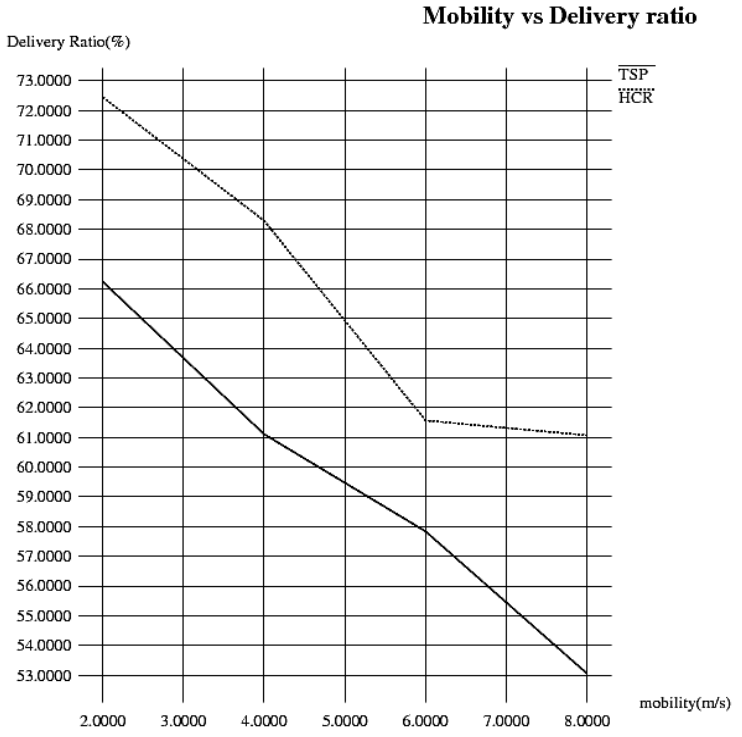

Fig. 7. Mobility VS delivery ratio.

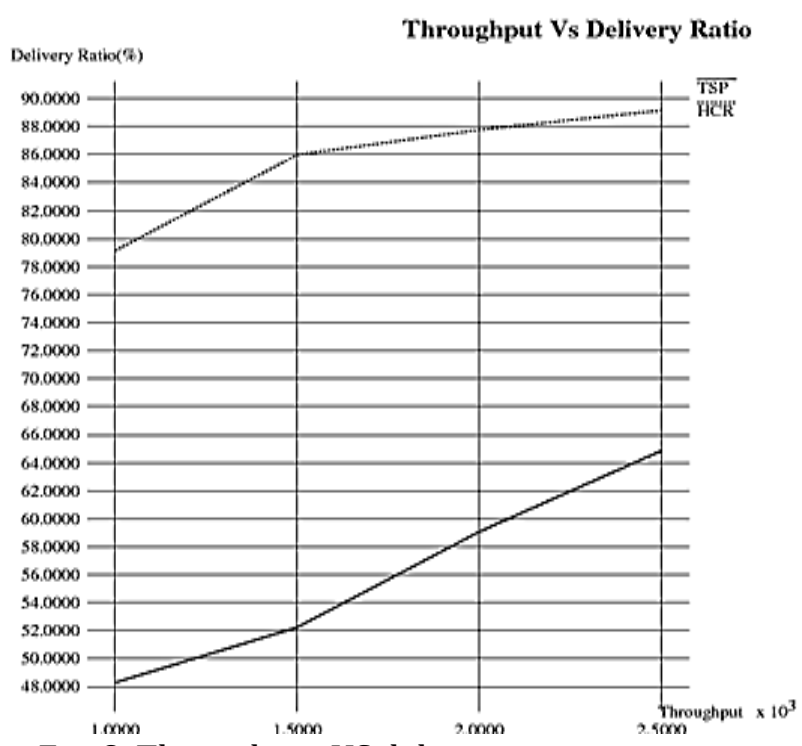

Fig. 8. Throughput VS delivery ratio.

Table 5. Throughput and Delivery Ratio

\begin{tabular}{|l|l|l|}
\hline Throughput & $\begin{array}{l}\text { Delivery Ratio (\%) of } \\
\text { TSP }\end{array}$ & $\begin{array}{l}\text { Delivery Ratio (\%) of the Proposed approach } \\
\text { (HCR) }\end{array}$ \\
\hline 1000 & 48.2865 & $\mathbf{7 9 . 1 3 8 7}$ \\
\hline 1500 & 52.2411 & $\mathbf{8 5 . 9 8 8 6}$ \\
\hline 2000 & 59.0763 & $\mathbf{8 7 . 7 7 3 6}$ \\
\hline 2500 & 64.8712 & $\mathbf{8 9 . 1 7 0 5}$ \\
\hline
\end{tabular}

The Fig. 8 illustrates throughput in Honeycomb routing approach by comparing with the TSP and Table 5 shows the throughput and their corresponding delivery ratio for TSP and HCR approach. The throughput efficiency in delivering the number of packets, thereby high (89.5\%) whereas in TSP results in lesser transfer of data packets yields a lower delivery ratio $(65 \%)$.

\section{Conclusion}

In this research work, it is concluded that the link distribution between the nodes gets strengthened, thus by increasing the data rate as well as increasing higher bandwidth utilization. The dropping of data packets gets minimized by using the wait and bound technique of admission control algorithm in AODV. From the simulation analysis has exhibited highly optimized results based on quality oriented parameters like network throughput, delivery ratio, bandwidth utilization, and end to end delay. The HCR has illustrated the reduction in network congestion and the overall network throughput has also increased by $89 \%$. Thus the proposed honeycomb routing algorithm has shown a high potential mechanism for bandwidth utilization efficiency of $91 \%$ and achieves QoS in a mobile adhoc environment to a maximum extent.

\section{Acknowledgement}

The authors are grateful to the anonymous referees for their valuable comments and suggestions to improve the presentation of this paper. 


\section{References}

[1] Amir, K. A., \& Sahoo, G. (2015). Behavior based high performance protocol for MANET. Indian Journal of Science and Technology.

[2] Kumar, G. R. S., \& Sundaram, K. M. M. (2014). Queuing based load balancing approach for improving network performance in manet. International Journal of Inventions in Computer Science and Engineering.

[3] Saini, A., \& Vinod, K. (2013). Mobile ad-hoc network routing protocols: Comparative study. International Journal of Enhanced Research in Science Technology and Engineering.

[4] Dinesh, K. S. (2014). Routing overhead reduction and selection of stable Paths in manet. International Journal of Inventions in Computer Science and Engineering, 1(9).

[5] Arockiam, L., Calduwel, N. P., \& Isac, G. J. (2010). A quality of service strategy to optimize bandwidth utilization in mobile networks. International Journal on Network Security.

[6] Swati, K., \& Deepender, D. N. (2013). Bandwidth estimation to provide QoS routing in manet. International Journal of Innovative Technology and Exploring Engineering.

[7] Suganya, S., \& Palaniammal, S. (2011). A well-organized dynamic bandwidth allocation algorithm for manet. International Journal of Computer Applications.

[8] Gunasekaran, R., Uthariaraj, V. R., Rajesh, R., Kaarthikeyan, S., \& Aravind, S. (2008). Priority scheduling in mobile ad hoc networks with improved bandwidth utilization. Proceedings of the IEEE Canadian Conference on Electrical and Computer Engineering.

[9] Diakite, L. H., \& Yu, L. (2012). A new algorithm to improve the sharing of bandwidth. International Journal of Science and Research.

[10] Mynuddin, S. R., \& Sreenivasa, R. D. (2012). Optimal bandwidth allocation technique in IEEE 802.11e mobile ad hoc networks (manet). Proceedings of the Second International Conference on Computer Science, Engineering and Applications.

[11] Feng, C., Hong, Q. Z., \& Fang, Y. G. (2010). Available bandwidth in multirate and multihop wireless ad hoc networks. IEEE Journal on Selected Areas in Communications.

[12] Srinivasa, G. G. K., Sri, K. C. V., \& Kashyap, D. D. (2013). Wireless measurement scheme for bandwidth estimation in multihop wireless ad hoc network. Global Journal of Computer Science and Technology Network, Web. Security.

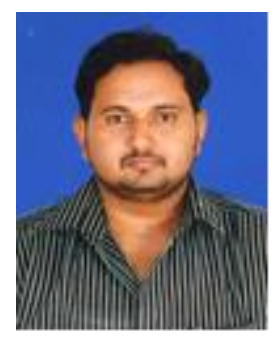

0. Cyril Mathew completed his undergraduate program in Nandha College of Engineering. He obtained his post graduate degree from Mahindra Engineering College and pursuing his Ph.D in Anna University, Chennai. At present he is working as an assistant professor in ECE Department in Al-Almeen Engineering College.

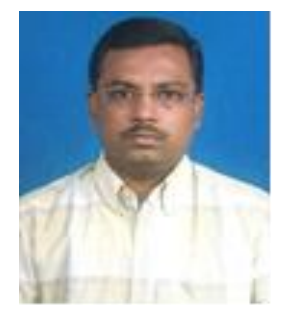

A. M. J. Zubair Rahman completed his undergraduate program and post graduate program in Bharathiyar University. He obtained his Ph.D degree from Anna University. At present he is working as a principal in Al-Almeen Engineering College. 